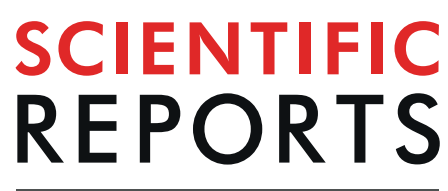

natureresearch

Check for updates

\title{
Long-term consequences of one anastomosis gastric bypass on esogastric mucosa in a preclinical rat model
}

\author{
Matthieu Siebert ${ }^{1}$, Lara Ribeiro-Parenti $\mathbb{1}^{1,3}{ }^{3}$, Nicholas D. Nguyen $\mathbb{D}^{1}$, Muriel Hourseau ${ }^{4}$, \\ Belinda Duchêne $\mathbb{D}^{5}$, Lydie Humbert ${ }^{6}$, Nicolas Jonckheere $\mathbb{D}^{5}$, Grégory Nuel ${ }^{7}$, \\ Jean-Marc Chevallier ${ }^{2}$, Henri Duboc ${ }^{1}$, Dominique Rainteau ${ }^{6}$, Simon Msika ${ }^{1,3}$, \\ Nathalie Kapel ${ }^{8}$, Anne Couvelard ${ }^{4}$, André Bado ${ }^{1}$ \& Maude Le Gall ${ }^{1}{ }^{1}$
}

\begin{abstract}
Although bariatric surgery is proven to sustain weight loss in morbidly obese patients, long-term adverse effects have yet to be fully characterized. This study compared the long-term consequences of two common forms of bariatric surgery: one-anastomosis gastric bypass (OAGB) and Roux-en-Y Gastric Bypass (RYGB) in a preclinical rat model. We evaluated the influence of biliopancreatic limb $(B P L)$ length, malabsorption, and bile acid (BA) reflux on esogastric mucosa. After 30 weeks of follow-up, Wistar rats operated on RYGB, OAGB with a short BPL (15 cm, OAGB-15), or a long BPL ( $35 \mathrm{~cm}$, OAGB-35), and unoperated rats exhibit no cases of esogastric cancer, metaplasia, dysplasia, or Barrett's esophagus. Compared to RYGB, OAGB-35 rats presented higher rate of esophagitis, fundic gastritis and perianastomotic foveolar hyperplasia. OAGB-35 rats also revealed the greatest weight loss and malabsorption. On the contrary, BA concentrations were the highest in the residual gastric pouch of OAGB-15 rats. Yet, no association could be established between the esogastric lesions and malabsorption, weight loss, or gastric bile acid concentrations. In conclusion, RYGB results in a better long-term outcome than $O A G B$, as chronic signs of biliary reflux or reactional gastritis were reported post-OAGB even after reducing the BPL length in a preclinical rat model.
\end{abstract}

Bariatric surgery is widely accepted as a long-term effective treatment for morbid obesity and ensuing metabolic disorders ${ }^{1}$. One anastomosis gastric bypass (OAGB) is a promising procedure, first reported in $1997^{2}$. This intervention has been proven safe ${ }^{2}$ with some studies even reporting a lower rate of post-operative morbidity compared to the gold standard, Roux-en-Y gastric bypass (RYGB) ${ }^{3,4}$. Additionally, OAGB may be preferred to RYGB due to its convenient ability to be reversed ${ }^{5}$ and revised ${ }^{6}$. Its efficiency in terms of weight loss and control of comorbidities has been widely characterized in the last several decades ${ }^{7-9}$, prompting its potential as an improved alternative to $\mathrm{RYGB}^{7}$.

However, OAGB is still debated due to chronic risks associated with potential biliary reflux ${ }^{10}$ on the esogastric tract.

OAGB, as all Omega-loop surgical strategies, is characterized by the direct anastomosis of the biliopancreatic loop to the stomach, instead of interposing an alimentary loop as in the Roux-en-Y procedures. This anatomically exposes the esogastric tract to bile acids (BA). In rats, the negative consequences of Omega-loop surgical strategies are well known ${ }^{11,12}$. Esojejunal or esoduodenal anastomoses have been reported as experimental models of

${ }^{1}$ Inserm UMRS 1149, UFR de Médecine Paris Diderot, Université de Paris, AP-HP, Paris, France. ${ }^{2}$ Department of digestive Surgery, AP-HP, Hôpital Européen Georges Pompidou, Paris, France. ${ }^{3}$ Department of General and Visceral Surgery, AP-HP, Bichat-Claude Bernard Hospital, Paris, France. ${ }^{4}$ Department of anatomopathology, AP-HP, BichatClaude Bernard Hospital, Paris, France. ${ }^{5}$ Univ. Lille, CNRS, Inserm, CHU Lille, UMR9020 - UMR-S 1277 - Canther Cancer Heterogeneity, Plasticity and Resistance to Therapies, Lille, France. ${ }^{6}$ Inserm UMR 7203, AP-HP Saint Antoine hospital, Paris, France. ${ }^{7}$ Stochastics and Biology Group (MAV), Probability and Statistics (LPSM), CNRS 8001, Sorbonne Université, Paris, France. ${ }^{8}$ Laboratoire de Coprologie Fonctionnelle, Hôpital Pitié-Salpêtrière Charles Foix, AP-HP, Paris, France. ${ }^{\bowtie}$-mail: Maude.le-gall@inserm.fr 
induced esophageal carcinogenesis ${ }^{12-14}$. Additionally in humans, Billroth II anastomosis, used for reconstruction after gastric cancer or gastric ulcer surgery, is associated with an increased risk of esogastric metaplasia and cancer compared to Roux-en-Y reconstruction ${ }^{15-17}$. A physiological hypothesis for these phenomena is that BA reflux could be responsible for chronic inflammation and oxidative stress - two major factors in the initiation of esophageal intestinal metaplasia and the eventual progression to adenocarcinoma ${ }^{18,19}$. Accordingly the first two cases of adenocarcinoma of the esophagogastric junction (AEG) following OAGB have been recently reported; the first reported a carcinoma of the gastric cardia (AEG II) ${ }^{20}$ and the second reported an adenocarcinoma of the esophagus (AEG I) $)^{21}$. However, it is important to note that in both cases patients were suffering from chronic reflux before and after the surgery.

Another point of debate is the impact of nutritional status on carcinogenesis. On one hand, epidemiological studies have revealed a strong link between obesity and cancer ${ }^{22}$. On the other, experimental studies have demonstrated that a lack of folate ${ }^{23}$, magnesium ${ }^{24}$, or vitamin $\mathrm{D}^{25}$ could act as co-factors in driving human digestive carcinogenesis. Broadly speaking, it is well known that bariatric surgery, particularly OAGB, may be responsible for these deficiencies ${ }^{26-28}$ or undernutrition ${ }^{29}$.

While OAGB has been performed for over 20 years, little objective data have described its long-term effects. To our knowledge, few cases of gastric cancer have been reported after omega-loop gastric bypass, an operation following similar principles to those of OAGB. However, three of these cancers were located in the region of the stomach excluded from the alimentary tract and only one of these cancers was located in the gastric pouch ${ }^{10}$. As of yet, only two published cases of esophageal or gastric cancer in patients post-OAGB have been published ${ }^{20,21}$ but there are no published long-term endoscopic studies. We previously explored the middle-term consequences of OAGB-induced biliary reflux on rats after 16 weeks and did not report an increased risk for esogastric cancer ${ }^{30}$.

The objective of this study was to evaluate long-term physiological consequences of OAGB on esogastric mucosa using a validated experimental model. OAGB rats were primarily compared to the gold standard RYGB and we hypothesized that OAGB rats were at a higher risk for post-operative precancerous or cancerous lesions. In order to better characterize the potential for adverse carcinogenic side effects in the esogastric physiology, we also analyzed the impact of the OAGB biliopancreatic limb (BPL) length on mucosal inflammation, BA concentration in the gastric pouch, and malabsorption after long-term follow-up.

\section{Results}

A schematic view of the experimental plan is presented in Supplementary Fig. S1.

Body weight, food intake, and caloric loss after bariatric surgery in lean animals. As previously observed, all bypass surgeries induced a rapid although transient weight loss, and maximal weight loss was observed between 10 and 14 days after surgery (Fig. 1A). The OAGB-35 group experienced the greatest weight loss, about 2-fold that experienced by the OAGB-15 and RYGB groups (OAGB-35 $-15.6 \pm 2.7 \%$ vs. OAGB-15 $-7.9 \pm 1.2 \%$ vs. RYGB $-9.0 \pm 1.1 \%$ ) (Fig. 1B). As a baseline control, we compared operated rats to unoperated control (CTRL) rats, and as expected, the CTRL group experienced the lowest observed weight loss $(-4 \pm 0.4 \%)$ (Fig. 1B). In the operated groups, weight loss was due to both reduced food intake and increased fecal caloric losses (Fig. 1C,D). Accordingly, daily caloric losses (Fig. 1E) during the second postoperative week were statistically more important in the OAGB-35 group ( $56.8 \pm 3.2 \%$ of calorie intake) compared to all groups, CTRL rats $(32 \pm 1.3 \%, P<0.0001)$, RYGB $(39.2 \pm 2.9 \%, P<0.001)$ and OAGB-15 $(40.9 \pm 2.4 \%, P<0.01)$.

We operated on non-obese young rats and followed them for 30 weeks, finding that all rats eventually regained weight over time. However, it is important to note that operated animals presented delayed weight gain compared to unoperated controls (Fig. 1A). After 30 weeks, all groups almost doubled their weight (CTRL: 186 $\pm 4.6 \%$; RYGB: $178 \pm 6.7 \%$; OAGB-15: $193 \pm 4.7 \%$; OAGB-35: $170 \pm 7.7 \%)$. Rats experienced almost $12 \%$ less weight gain post OAGB-35 than post OAGB-15 $(P<0.05)$. Although not statistically significant, OAGB-35 rats experienced almost $7 \%$ less weight gain than the RYGB rats.

Esophagus and gastric lesions after bypass surgeries. To analyze putative esophageal lesions resulting from the different surgeries, HES-staining of esophageal mucosa were analyzed (Fig. 2 and Supplementary Fig. S2) and scored as either healthy esophageal mucosa (HEM, Fig. 2A), esophageal hyperpapillomatosis (EHP, Fig. 2B), or esophagitis (Fig. 2C). Surprisingly, unoperated control animals displayed more esophageal lesions than RYGB animals ( $50 \%$ of CTRL, $n=4 / 8$ vs. $10 \%$ of RYGB, $n=1 / 10)$. Specifically, EHP was observed in only $1 / 10$ rat from the RYGB group with no occurrence of esophagitis whereas $4 / 8$ rats in the unoperated group showed signs of EHP with $1 / 8$ experiencing esophagitis.

Histological lesion occurrence in the OAGB-15 group was close to that of the unoperated group with $60 \%$ of the samples experiencing HEM $(n=6 / 10) ; 30 \%$ experiencing EHP $(n=3 / 10)$; and $10 \%$ experiencing esophagitis $(n=1 / 10)$. The OAGB-35 group had the highest percentage of esophagitis $(50 \% ; n=5 / 10)$ and the lowest percentage of HEM $(30 \%, n=3 / 10)$ compared to the other groups.

Esophageal anatomopathology was analyzed using Fisher exact test to determine statistical significance (Supplementary Tables 1-4 and Supplementary Text 1). The percentage of observed esophageal lesions was statistically different between groups $(P<0.001)$. Rats that underwent RYGB displayed a lower percentage of esophageal lesions when compared to unoperated rats although the difference was not significant $(P=0.157)$. With the study design, the power (at level 5\%) for the corresponding comparison is 0.352 suggesting that the non-significance might be due to the low power of the study. Most importantly, there was a prominent increase in the percentage of esophageal lesions present in OAGB-35 when compared to RYGB $(P=0.008)$.

In order to evaluate changes in the fundic mucosa, we characterized the histological slices as having either healthy gastric mucosa (HGM, Fig. 3A-C) or foveolar hyperplasia (FH, Fig. 3D-F). The fundic mucosa was 
A

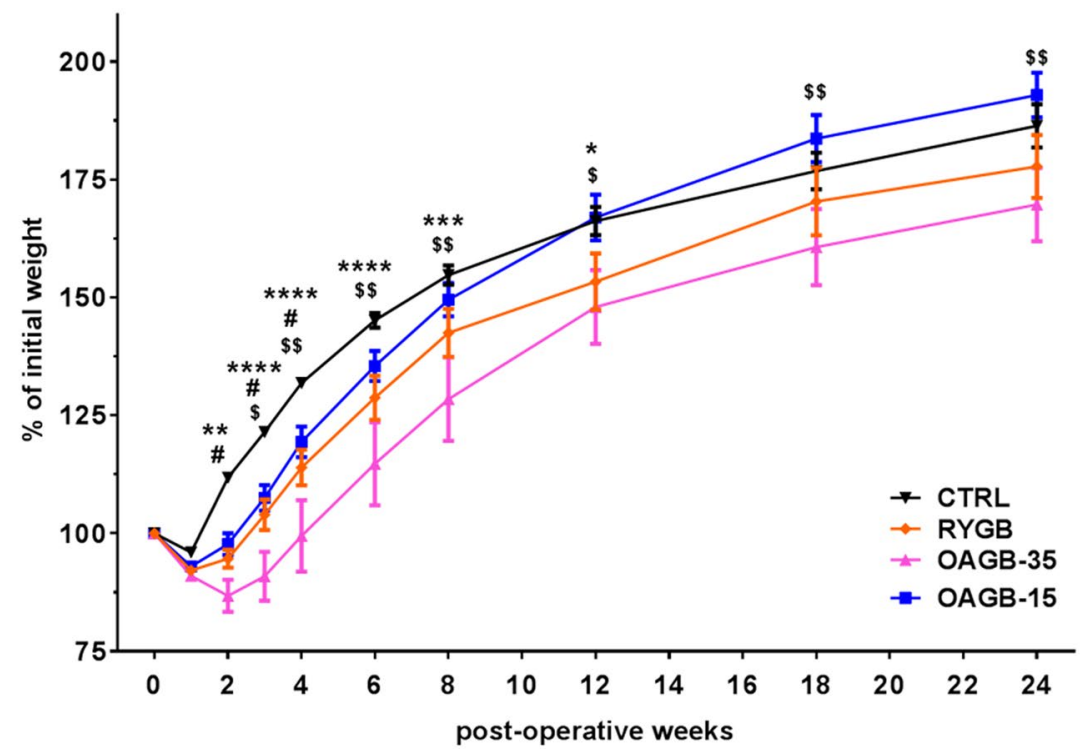

B

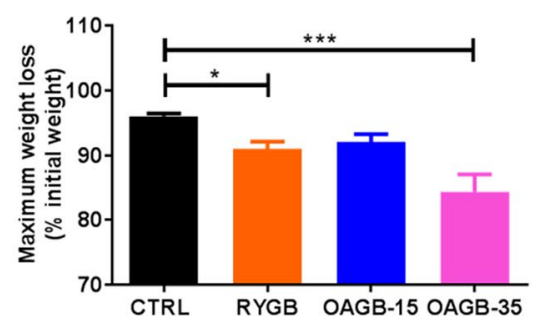

D

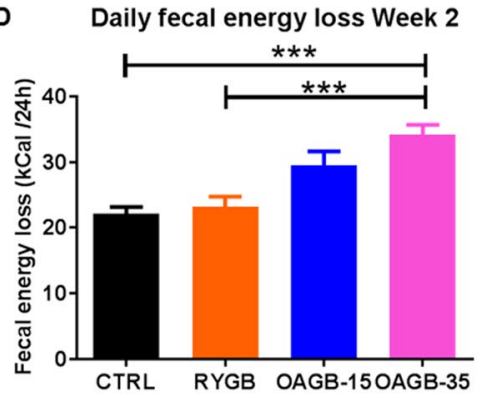

C Daily food intake Week 2

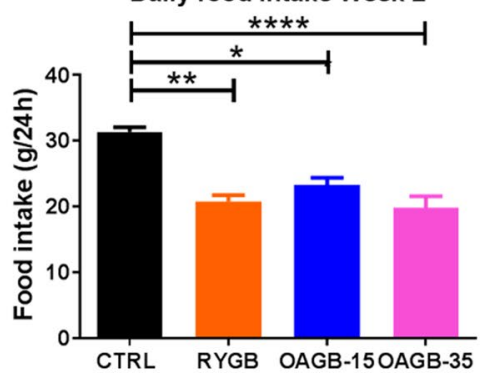

E Daily caloric loss Week 2

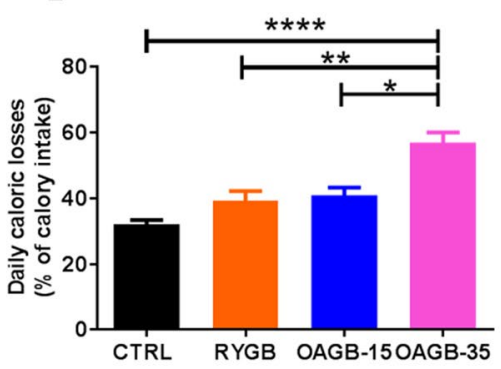

Figure 1. Gastric Bypass surgery in lean rats induced a delay in weight gain and OAGB-35 is associated with reduced food intake and increased malabsorption. (A) Weight loss and regain according to surgical procedure during 24 post-operative weeks. $* P<0.05$; $* * * P<0.001$, $* * * * P<0.0001$ OAGB-35 vs. unoperated CTRL. ${ }^{\#} P<0.05$ RYGB vs. unoperated CTRL. ${ }^{\$} P<0.05 ;{ }^{\$ \$} P<0.01$ OAGB-35 vs. OAGB-15 by Tukey’ multiple comparison tests after 2-way ANOVA. (B) Maximum weight loss according to surgical procedure, (C) daily food intake, (D) fecal energy loss and, (E) caloric loss during the second postoperative week. $* P<0.05$; $* * P<0.01 ; * * * P<0.001, * * * * P<0.0001$ by Dunn's multiple comparison tests after Kruskal Wallis test.

analyzed in all animals (Fig. 3G), and for those that underwent gastric bypass, the region of the gastro-jejunal anastomosis was also studied (Fig. 3H). No precancerous or cancerous lesions were observed.

The OAGB-35 group presented the highest number of altered mucosa with $60 \%(n=6 / 10) \mathrm{FH}$ in the fundic mucosa as in the region of the gastro-jejunal anastomosis while RYGB presented only $10 \%(\mathrm{n}=1 / 10)$ FH in both regions. Statistical analyses of fundic and perianastomotic mucosa anatomopathology (Supplementary Table 2-4 and Supplementary Text 1 ) revealed that the percentage of gastropathy was close to being statistically different between RYGB and OAGB-35 ( $P=0.0573$ for both fundic and perianastomotic mucosa). The corresponding power at a level of $5 \%$ is 0.436 for fundic and 0.444 for perianastomotic mucosa. However, the combination of both tables reveals a $P$-value of 0.0137 confirming the fact that lesions in the fundic and perianastomotic mucosa after OAGB-35 were more important than after RYGB. 

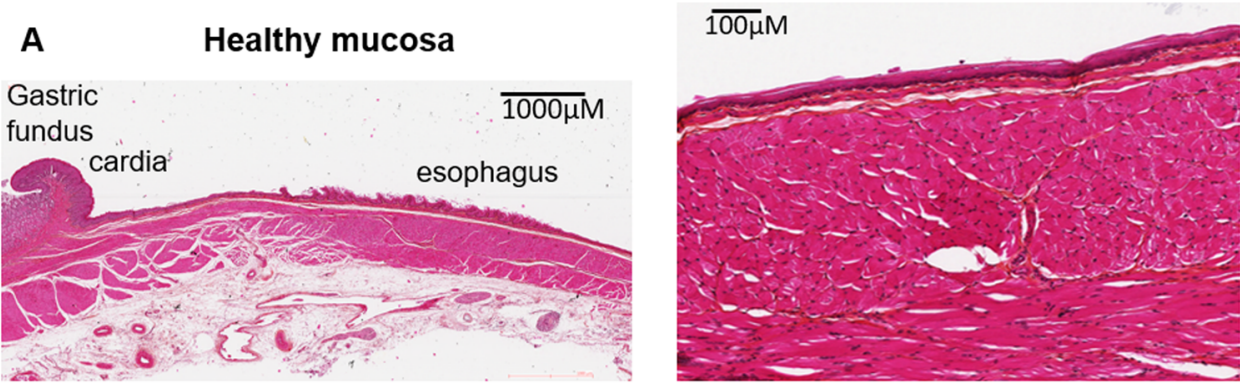

B Hyperpapillomatosis
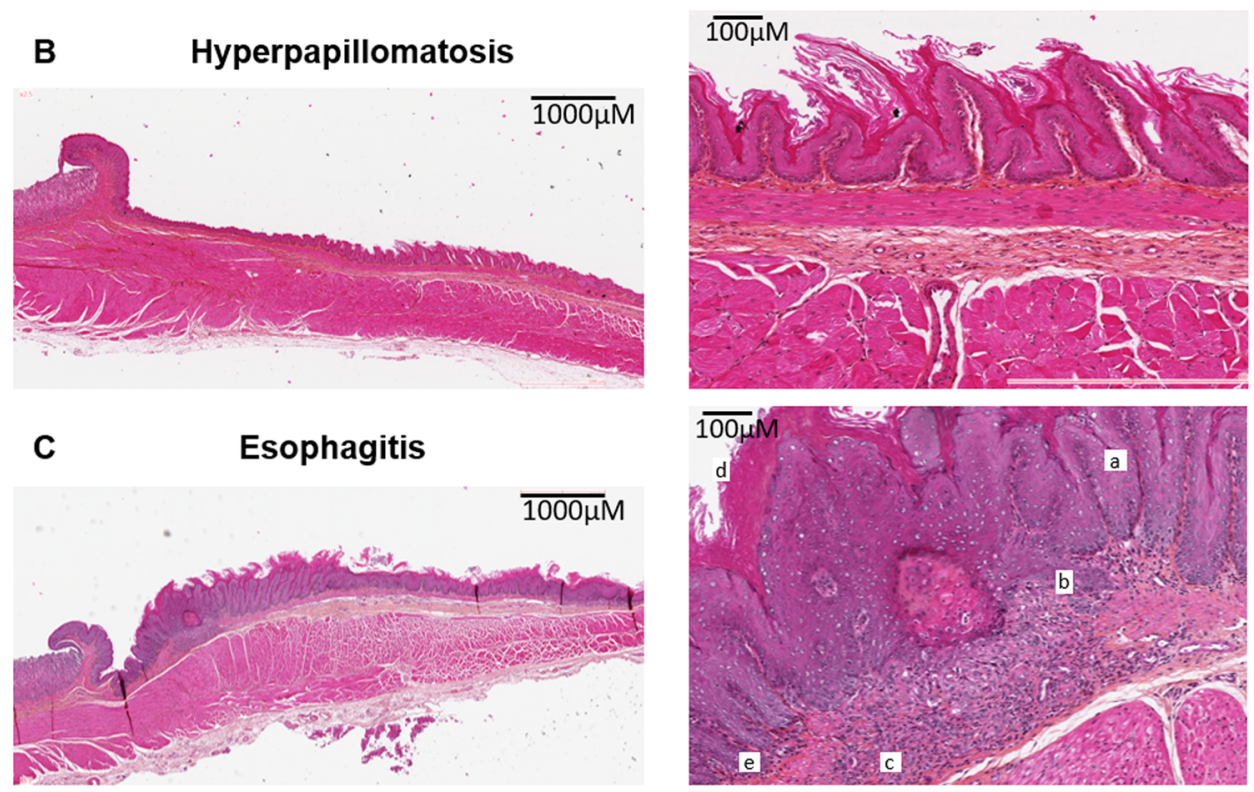

\section{Esophageal anatomopatholgy}

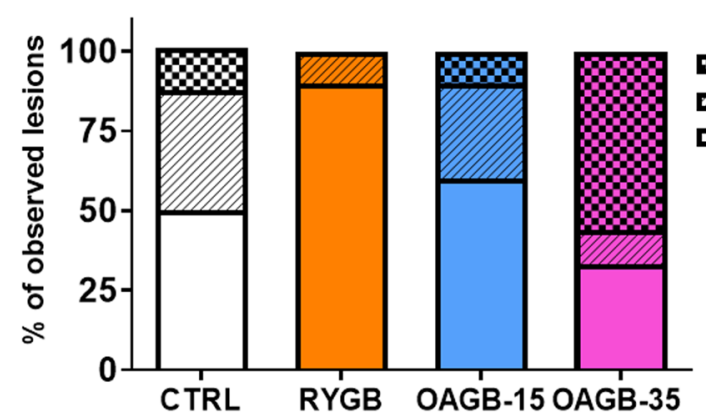

\section{Esophagitis \\ Hyperpapillomatosis \\ ㅁealthy mucosa}

Figure 2. One-anastomosis gastric bypass with extended biliopancreatic limb increases risk for esogastric lesions. (A-C) Representative HES staining of healthy esophageal mucosa (A), esophageal hyperpapillomatosis (B) and esophagitis (C) illustrating an association with hyperpapillomatosis (a), basal cell proliferation (b), fibrosis (c), hyper ortho-keratosis (d), and immune cell infiltration (e). (D) Quantification of esophageal lesions according to surgical procedure and expressed as a percent. CTRL $n=8$, RYGB $n=10$, OAGB-15 $n=10$ and OAGB-35 $\mathrm{n}=10$.

We complemented these anatomopathological analyses by performing immunohistochemistry, probing for Mucin 2, Mucin 4 and Mucin 5B (Supplementary Fig. S2) as putative markers of Barrett's esophagus. Compared to healthy mucosa, no additional staining was observed in hyperpapillomatosis or esophagitis lesions from either the esophagus or fundus of the stomach, suggesting the absence of Barrett's esophagus.

Gastric biliary acid content. Biliary acid (BA) reflux within the gastric pouch has been a controversial post-operative long-term consequence of OAGB. To gain a better understanding of the effects of gastrointestinal modifications post-bariatric surgery, we measured total BA, primary BA, and secondary BA within the stomachs of unoperated CTRL animals and the residual gastric pouches of operated rats (Fig. 4A-C). 
A Healthy mucosa

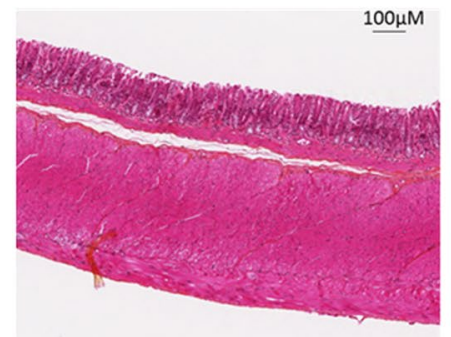

D Foveolar hyperplasia

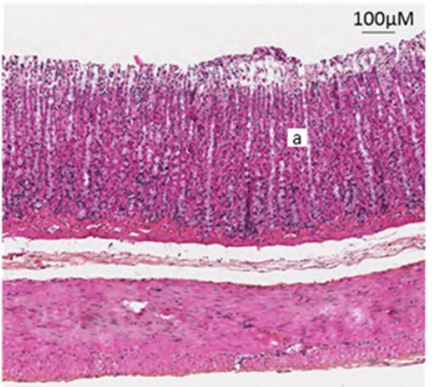

G
B

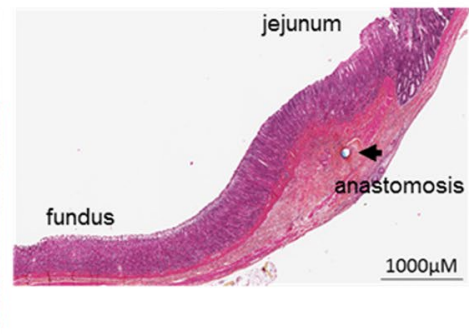

E

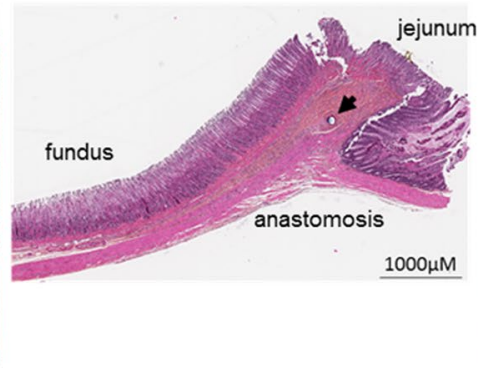

C

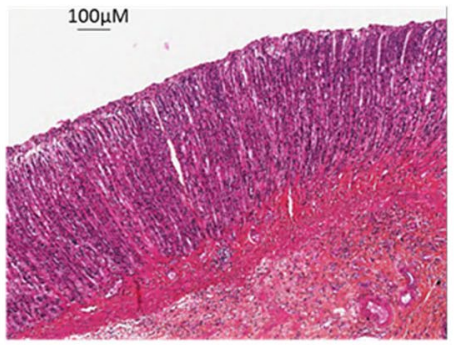

$\mathbf{F}$

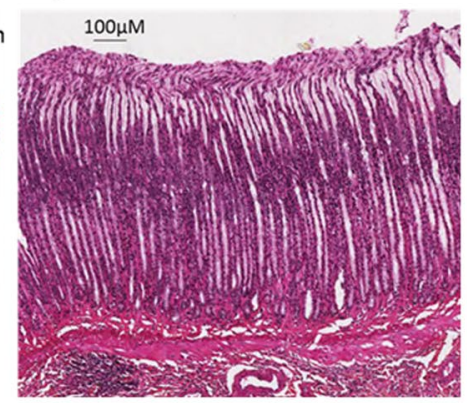

H Perianastomotic anatomopathology
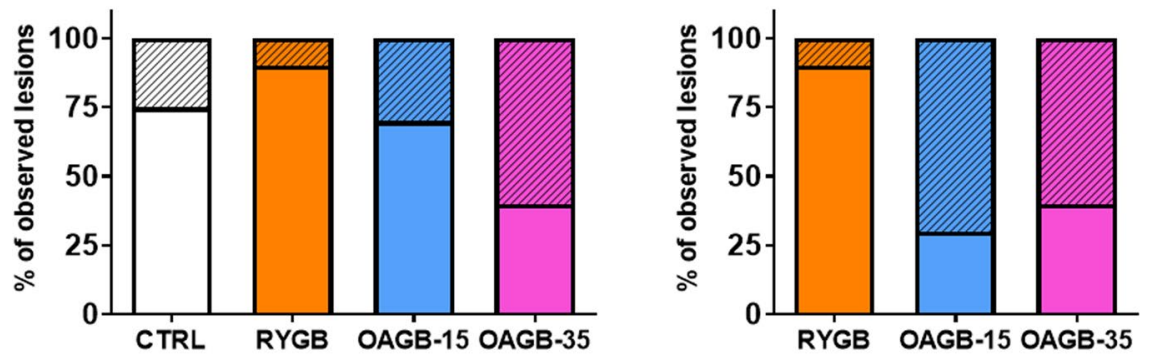

Foveolar

myperplasia

Healthy

mucosa

Figure 3. OAGB is associated with elevated perianastomotic and fundic foveolar hyperplasia. (A-C) Representative HES staining of a healthy mucosa next to a gastrojejunal anastomosis (arrow points to the anastomosis). (D,E) Representative HES staining of (D) fundic and (F) perianastomotic foveolar hyperplasia displaying parietal cell proliferation (a). (G,H) Quantification of gastric anatomopathological lesion in the fundus $(\mathbf{G})$ and near the anastomosis $(\mathbf{H})$ expressed as a percent. CTRL $n=8$, RYGB $n=10$, OAGB-15 $n=10$ and OAGB-35 $n=10$.

Detailed statistical analyses (Supplementary Table 5 and Supplementary Text 2) shows that total, primary and secondary BA concentrations were not statistically different between CTRL and RYGB animals $(P=0.649$, $P=0.779$, and $P=0.342$ RYGB vs. CTRL respectively). However total and primary BA concentrations were statistically different between the three operated groups $(P=0.0504$ and $P=0.0375$ respectively). In particular, concentrations of total and primary BA were about 2.3 fold higher in OAGB-15 animals compared to RYGB animals (OAGB-15 $=2,536 \mu \mathrm{M}$ vs. $\mathrm{RYGB}=1,114 \mu \mathrm{M}, P=0.0279$ for total BA and OAGB- $15=1,909 \mu \mathrm{M}$ vs. $\mathrm{RYGB}=822 \mu \mathrm{M}, P=0.0271$ for primary $\mathrm{BA})$. No other statistical difference was highlighted; although, a trend towards increased levels of secondary BA was observed in the two OAGB groups in comparison to RYGB with $P=0.179$ and a power at level $5 \%$ of 0.356 .

To test whether gastric BA concentration is associated with esophageal or gastric lesions independent of surgical procedure, we pooled all subjects from each group and classified them based on anatomopathology (Fig. 4D-F). Considering all groups together, total BA gastric concentrations were not associated with an abnormal esophageal $(P=0.40)$, fundic $(P=0.59)$ or perianastomotic $(P=0.42)$ anatomopathology.

Undernutrition markers. We suspected that the OAGB-associated esogastric lesions were linked to undernutrition. To test whether weight loss was associated with abnormal esophageal anatomopathology, rats were compared according to their maximal weight loss but independent of surgical procedure. An intriguing observation was that hyperplasia was associated with a lower weight loss compared to healthy esophageal mucosa or esophagitis (Fig. 4G). The development of esophagitis on the other hand did not appear to correlate with weight loss across all groups. Similarly, weight loss was not associated with fundic or perianastomotic foveolar hyperplasia (Fig. 4H,I).

Plasma concentrations of creatinine, urea, albumin, protein, calcium, phosphates, Vitamin D, iron, ferritin, transferrin, triglycerides, total and HDL cholesterol, NEFA, ALAT, and ASAT were assayed to determine whether animals were undernourished 30 weeks after surgery (Table 1). Although OAGB-35 animals display abnormal 

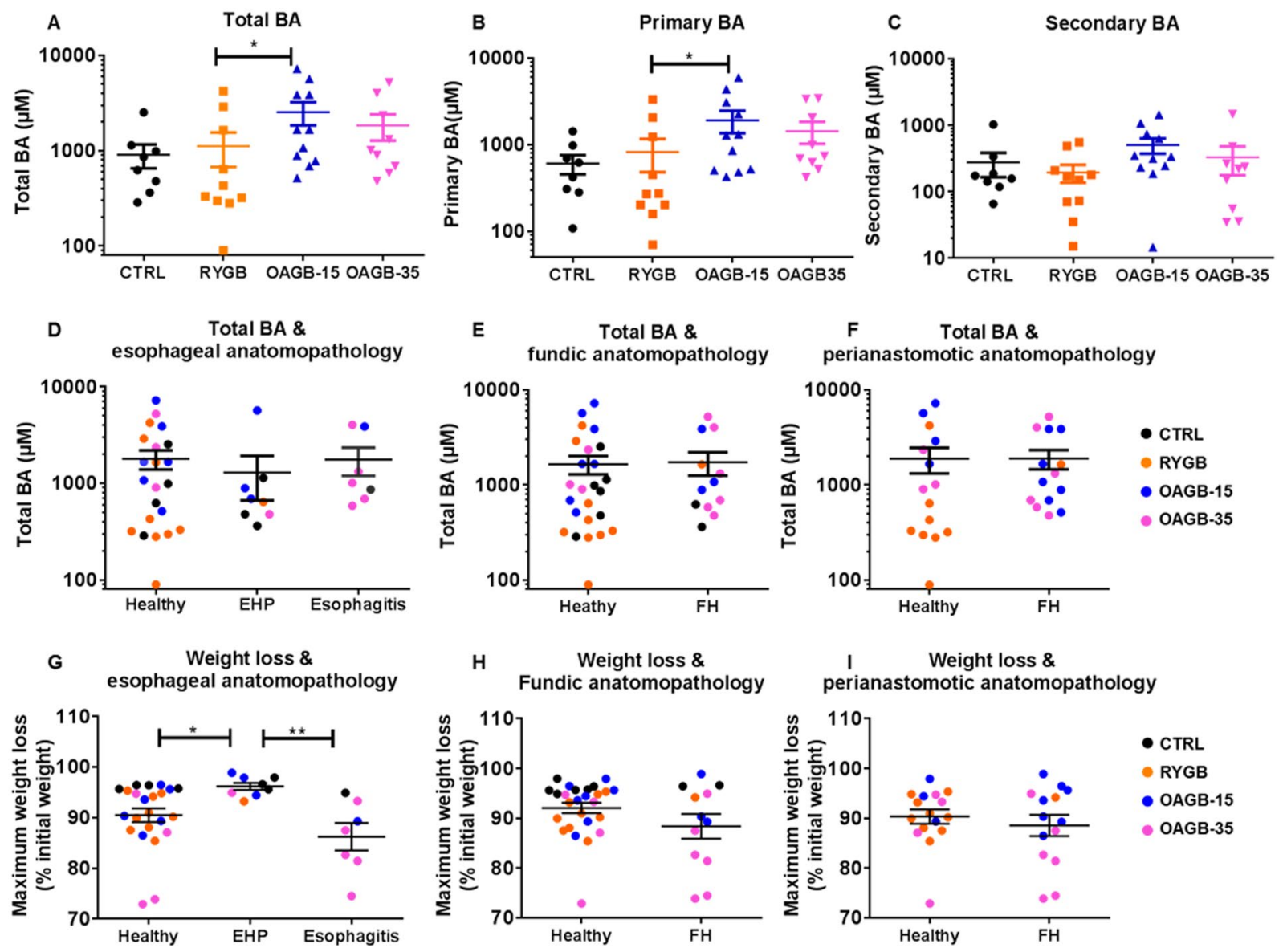

Figure 4. Bile acid concentrations are higher in the gastric pouch of OAGB groups but not associated with esogastric pathologies. (A) Total, (B) primary and (C) secondary biliary acid (BA) concentration $(\mu \mathrm{M})$ within the stomach (CTRL) and remaining gastric pouch (post-gastric bypass) according to surgical procedure. Esophageal (D), fundic (E), and perianastomosis (F) anatomopathology according to BA concentrations. Esophageal (G), fundic $(\mathbf{H})$ and perianastomotic (I) anatomopathology according to maximum weight loss. (CTRL in black, RYGB in orange, OAGB- 15 in blue, OAGB-35 in pink). $* P<0.05$ by Dunn's multiple comparison tests after Kruskal Wallis test. Abbreviations: EHP: esophageal hyperpapillomatosis; FH: Foveolar Hyperplasia.

values compared to unoperated CTRL rats for some of these markers (notably higher uremia and lower vitamin D) no association between anatomical lesions and biochemical parameters were discerned.

\section{Discussion}

Following 30 weeks post-OAGB and -RYGB, we showed that no rats displayed signs of metaplasia, dysplasia, Barrett's esophagus, or esogastric cancer. We must note however that OAGB-35 rats experienced higher rates of esophagitis in addition to fundic and perianastomotic foveolar hyperplasia compared to the RYGB group. Furthermore, although we were not able to establish any association between the presence of lesions and either malabsorption, weight loss, or gastric bile acid concentrations, we did notice that OAGB rats with a long BPL experienced the greatest malabsorption and highest incidence of esogastric lesions.

Patients that undergo bariatric surgery are fairly young with a mean age of $42^{31}$. Based on the WHO's Global Health Observatory data in 2016, these patients are expected to live another 30 years with worldwide life expectancy averaging 72 years $^{32}$. As such, they are at risk for long-term side effects that could arise as the result of these procedures. This preclinical OAGB study presents a longitudinal study with a 30 weeks follow-up in rats that is equivalent to 22-30 years of human life ${ }^{33}$. In order to maintain clinical relevance, we compared OAGB to the gold standard RYGB, and we used rats that did not undergo any operation as a control group to recapitulate patients without bariatric surgery.

As a result of the anatomical remodeling of the gastric pouch, risks of biliary reflux and resulting long-term complications still leave OAGB a controversial procedure today. Our study provides evidence that the risk of esophageal cancer after OAGB remains a possibility after observing FH and esophagitis lesions. Even though FH is a sign of chemical gastritis and cannot be strictly interpreted as precancerous lesions, physiological mechanisms leading to FH could be similar to those leading to esophageal lesions. The presence of these lesions signify the earliest step of the esophageal carcinogenic sequence. Involvement of BA is suggested by the occurrence of FH at about $60 \%$ in the OAGB groups compared to $10 \%$ in RYGB group. The complex role of biliary reflux as either an unassociated independent factor or as a potentializing factor of pre-existent gastro-esophageal reflux (GER) has 


\begin{tabular}{|c|c|c|c|c|c|c|}
\hline \multicolumn{2}{|c|}{ Plasma concentrations } & \multirow{2}{*}{\begin{tabular}{|l|} 
CTRL \\
53 \\
\end{tabular}} & \multirow{2}{*}{$\begin{array}{l}\text { RYGB } \\
52.4\end{array}$} & \multirow{2}{*}{\begin{tabular}{|l|} 
OAGB 15 \\
54.4
\end{tabular}} & \multirow{2}{*}{$\begin{array}{l}\text { OAGB } 35 \\
50.6\end{array}$} & \multirow{2}{*}{ 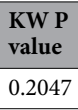 } \\
\hline Creatinine & Mean & & & & & \\
\hline \multirow[t]{2}{*}{$(\mu \mathrm{mol} / \mathrm{L})$} & SEM & 1.461 & 1.424 & 1.352 & 0.8718 & ns \\
\hline & Stats & & ns & ns & $\mathrm{ns}$ & \\
\hline Urea & Mean & 4.583 & 5.61 & 5.3 & 5.86 & 0.0007 \\
\hline \multirow[t]{2}{*}{$(\mathrm{mmol} / \mathrm{L})$} & SEM & 0.08724 & 0.2942 & 0.09145 & 0.1845 & $* * *$ \\
\hline & Stats & & $*$ & $*$ & $* * *$ & \\
\hline Albumin & Mean & 26.89 & 24.19 & 26.33 & 25.76 & 0.0271 \\
\hline \multirow[t]{2}{*}{$(\mathrm{g} / \mathrm{L})$} & SEM & 0.8081 & 0.5381 & 0.4206 & 0.6318 & $*$ \\
\hline & Stats & & $*$ & $\mathrm{~ns}$ & ns & \\
\hline Protein & Mean & 54.01 & 50.73 & 54.9 & 54.65 & 0.0331 \\
\hline \multirow[t]{2}{*}{$(\mathrm{g} / \mathrm{L})$} & SEM & 0.642 & 1.104 & 0.9747 & 0.9507 & $*$ \\
\hline & Stats & & ns & ns & $\mathrm{ns}$ & \\
\hline Calcium & Mean & 2.447 & 2.343 & 2.364 & 2.368 & 0.0674 \\
\hline \multirow[t]{2}{*}{$(\mathrm{mmol} / \mathrm{L})$} & SEM & 0.01742 & 0.0206 & 0.0201 & 0.03524 & ns \\
\hline & Stats & & $*$ & ns & ns & \\
\hline Phosphate & Mean & 2.146 & 2.08 & 2.341 & 2.215 & 0.0159 \\
\hline \multirow[t]{2}{*}{$(\mathrm{mmol} / \mathrm{L})$} & SEM & 0.07819 & 0.05434 & 0.04581 & 0.05689 & $*$ \\
\hline & Stats & & $\mathrm{ns}$ & ns & $\mathrm{ns}$ & \\
\hline Vitamin D & Mean & 64.95 & 49.4 & 54.87 & 41.51 & 0.0277 \\
\hline \multirow[t]{2}{*}{$(\mathrm{nmol} / \mathrm{L})$} & SEM & 4.877 & 4.723 & 2.917 & 5.059 & $*$ \\
\hline & Stats & & ns & ns & $* *$ & \\
\hline Iron & Mean & 26.96 & 12.35 & 18.2 & 13.84 & 0.003 \\
\hline \multirow[t]{2}{*}{$(\mu \mathrm{mol} / \mathrm{L})$} & SEM & 2.701 & 2.566 & 1.735 & 1.98 & $* *$ \\
\hline & Stats & & $* *$ & ns & $* *$ & \\
\hline Ferritin & Mean & 51.56 & 36.39 & 49.06 & 41.88 & 0.3415 \\
\hline \multirow[t]{2}{*}{$(\mathrm{ng} / \mathrm{mL})$} & SEM & 4.964 & 4.437 & 8.584 & 10.51 & $\mathrm{~ns}$ \\
\hline & Stats & & $\mathrm{ns}$ & ns & $\mathrm{ns}$ & \\
\hline Transferrin & Mean & 1.136 & 1.156 & 1.163 & 1.205 & 0.9743 \\
\hline \multirow[t]{2}{*}{$(\mathrm{g} / \mathrm{L})$} & SEM & 0.07393 & 0.03458 & 0.03462 & 0.06337 & ns \\
\hline & Stats & & $\mathrm{ns}$ & ns & $\mathrm{ns}$ & \\
\hline Triglycerides & Mean & 0.79 & 0.8444 & 0.7045 & 0.858 & 0.6756 \\
\hline \multirow[t]{2}{*}{$(\mathrm{mmol} / \mathrm{L})$} & SEM & 0.09256 & 0.12 & 0.07453 & 0.0964 & ns \\
\hline & Stats & & $\mathrm{ns}$ & ns & $\mathrm{ns}$ & \\
\hline \multirow[t]{3}{*}{$\begin{array}{l}\text { Total Cholesterol } \\
(\mathrm{mmol} / \mathrm{L})\end{array}$} & Mean & 3.491 & 1.981 & 2.083 & 2.09 & 0.0037 \\
\hline & SEM & 0.5467 & 0.1093 & 0.112 & 0.1482 & $* *$ \\
\hline & Stats & & $* *$ & ** & $*$ & \\
\hline \multirow[t]{3}{*}{$\begin{array}{l}\text { HDL Cholesterol } \\
(\mathrm{mmol} / \mathrm{L})\end{array}$} & Mean & 1.637 & 0.835 & 0.9873 & 0.986 & 0.0029 \\
\hline & SEM & 0.2435 & 0.07046 & 0.07289 & 0.08543 & $* *$ \\
\hline & Stats & & **** & $*$ & $*$ & \\
\hline NEFA & Mean & 1.064 & 0.5111 & 0.6336 & 0.414 & 0.0605 \\
\hline \multirow[t]{2}{*}{$(\mathrm{mmol} / \mathrm{L})$} & SEM & 0.2308 & 0.1806 & 0.2135 & 0.07317 & ns \\
\hline & Stats & & $*$ & ns & $\mathrm{ns}$ & \\
\hline ALAT & Mean & 45.86 & 52.44 & 64.64 & 56.6 & 0.0288 \\
\hline \multirow[t]{2}{*}{$(\mathrm{U} / \mathrm{L})$} & SEM & 3.801 & 7.485 & 3.781 & 4.655 & $*$ \\
\hline & Stats & & ns & $*$ & ns & \\
\hline ASAT & Mean & 117.1 & 98 & 167.8 & 147.4 & 0.0618 \\
\hline$(\mathrm{U} / \mathrm{L})$ & SEM & 9.585 & 7.885 & 23.85 & 18.17 & ns \\
\hline & Stats & & ns & ns & ns & \\
\hline
\end{tabular}

Table 1. Main biochemical parameters measured 30 weeks after surgery. Mean and Standard error of mean $(\mathrm{SEM})$ of plasma concentrations of creatinine $(\mu \mathrm{mol} / \mathrm{L})$, urea $(\mathrm{nmol} / \mathrm{L})$, albumin $(\mathrm{g} / \mathrm{L})$, protein $(\mathrm{g} / \mathrm{L})$, calcium $(\mathrm{mmol} / \mathrm{L})$, phosphates $(\mathrm{mmol} / \mathrm{L})$, vitamin $\mathrm{D}(\mathrm{nmol} / \mathrm{L})$, iron $(\mu \mathrm{mol} / \mathrm{L})$, ferritin $(\mathrm{ng} / \mathrm{mL})$, transferrin $(\mathrm{g} / \mathrm{L})$, triglycerides $(\mathrm{mmol} / \mathrm{L})$, total and HDL cholesterol $(\mathrm{mmol} / \mathrm{L})$, Non esterified Fatty Acids (NEFA) (mmol/L), Alanine Aminotransferase (ALAT) (U/L), and Aspartate Aminotransferase (ASAT) (U/L). Comparison between groups was conducted using Kruskal Wallis (KW) non-parametric tests followed by Dunn's multiple comparisons test (Stats) to compare bypass with CTRL. $* P<0.05 ; * * P<0.01 * * * P<0.001$. 
been suggested by several studies ${ }^{14,34}$. Yet, in our present study, residual gastric pouch BA concentrations were not associated with the occurrence of anatomopathological lesions. This interesting finding suggests that BA exposure to esophageal mucosa may not by itself promote esogastric anatomopathological lesions. Furthermore, a longer BPL was associated with an increased rate of esophageal lesions compared to a shorter BPL despite a trend toward lower gastric pouch BA concentrations in the long BPL group. This observation runs against the notion that a longer BPL could decrease the risk of esophagitis due to partial reabsorption of $\mathrm{BA}^{35}$. We hypothesize that the nutritional status, notably undernutrition, could be a co-factor in esophageal lesions that incite carcinogenesis. It is well known that gastric bypass may lead to micronutrient deficiencies ${ }^{28,29,36}$. OAGB is suspected to be associated with an increased sarcopenic undernutrition ${ }^{37}$, and a longer BPL is known to increase nutritional complication and undernutrition ${ }^{28,38}$. Accordingly, the OAGB rats with the long BLP experienced a drastic initial weight loss, a concurrent lower weight gain at the end of the experiment and reduced vitamin D plasma levels. OAGB rats with the long BLP were also associated with the highest occurrence of esophagus lesions. Undernutrition and micronutrient deficiencies could thus weaken the esophageal mucosa against aggressions secondary to biliary reflux, overpowering the beneficial features of OAGB and increasing the chances for carcinogenesis.

A paradoxical observation we reported was the association of reduced weigh loss with esophageal hyperpapillomatosis but not with esophagitis. One could hypothesize this to be a physiological adaptation promoting nutrient absorption in food-depleted animals as already reported in Short Bowel Syndrome (SBS) animal models ${ }^{39}$. However, in the SBS model of undernutrition, hyperplasia has been described in the colon and jejunum, whereas the esophagus was not studied. Transposition of jejunum within the esophagus has been reported to induce hyperplasia of the transposed jejunum ${ }^{40}$. Nevertheless, to the best of our knowledge, study of the adaptation of the esophagus (hyperplasia and/or hyperpapillomatosis) and its contribution to nutrient absorption in undernourished animal models has never been published and merit further attention.

Despite the fact that long-term safety of OAGB has not been unequivocally established, it appears that specific characteristics of OAGB and RYGB could protect the esophageal mucosa against biliary reflux consequences. Rats are recognized as a good model of esophageal carcinogenesis and a promising model to reproduce the human carcinogenic sequence ${ }^{13,41}$. In other experimental studies, esojejunal anastomosis on rats led to Barrett's esophagus in $92 \%$ of cases after 30 weeks and cancer rate up to $8 \%$ without any co-carcinogen in F344 rats ${ }^{42}$ and esophageal intestinal metaplasia and mucosal ulcerations were observed in $41.7 \%$ and $50 \%$ of wistar rats with esojejunal anastomosis ${ }^{43}$. These experiments and ours suggest putative protective factors specific to gastric bypass surgeries ${ }^{43}$. GER is a well-known risk factor of esophageal cancer ${ }^{34,44}$. Here, we propose the potential impact of GER on esophageal mucosa because unoperated rats presented a rate of esophageal hyperpapillomatosis (EHP) of 50\%, alleging that quadruped animals might be exposed to a physiological GER. In contrast, EHP lesions were almost absent in the RYGB group, revealing an inherent protective effect of a gastro-jejunal anastomosis on the esophageal mucosa. The gastro-jejunal anastomosis bypasses the pylorus, which likely leads to a reduction of acid production and gastric pressure. This hypothesis is supported by the positive results of the RYGB on GER disease symptoms and preservation of esophageal function in humans ${ }^{45}$. This supports why RYGB is considered a therapeutic option in the case of resistant GER disease $\mathrm{f}^{46,47}$. As for OAGB surgery, acidic gastric secretions produced in the gastric pouch could be neutralized by basic BA, reducing their agressivity ${ }^{13,48}$. In humans, but not in our experimental model, the OAGB's long and tubular gastric pouch should mechanically diminish exposure of the esophagus to gastric content in contrast to the small RYGB gastric pouch. In addition, weight loss post-bariatric surgery leads to a reduction of $\mathrm{GER}^{49}$. Altogether, these factors could explain why there is no case of esophageal cancer described in this experiment and why only one case was reported 20 years after the first human OAGB ${ }^{21}$.

We recognize that our study focuses on the anatomy of rats and still requires further investigation in order to be translated to human physiology. Rat esophageal histology is inherently different from humans due to the presence of a keratinized epithelium, not to mention quadrupeds naturally display esophageal and gastric lesions. In addition, the limited sample size resulted in a low power of most of our statistical analyses and prevented us from exploring whether there was an additive effect of BA and undernutrition in regards to the development of esogastric lesions. We must also note that unoperated animals as a baseline control may yield slightly different histological physiology than those that would have underwent a sham surgery. However, we found that this would most closely mirror patients without surgery. Our model of lean Wistar rats must also be taken into consideration when studying carcinogenesis, since obesity is a well-known risk factor of cancer ${ }^{50}$, especially esophageal cancer ${ }^{51}$. Nevertheless, this experimental design on lean animals allowed us to study individual procedure impact independent of obesity, preventing weight loss as a cofounding factor and revealing baseline effects of bariatric surgery on gastrointestinal physiology. Observing subtle changes in lean rats provides compelling concerns that biliary reflux could have an additive effect in cancer risk when coupled with obesity, and definitive conclusions would require further investigation in obese rats.

Most importantly, a clinical study exploring esogastric mucosa longitudinally after OAGB is needed to confidently eliminate the risk of cancer following this procedure. The recent finding that sleeve gastrectomy has been associated with a higher risk of Barrett's esophagus ${ }^{52}$ provides a compelling reason for repeated endoscopic exploration after surgery ${ }^{53}$. Until longitudinal endoscopic studies post-surgery are conducted to characterize the negative impact of OAGB on esogastric mucosa, endoscopic follow-up is crucial in determining whether or not carcinogenicity can be ruled out.

\section{Materials and Methods}

Ethics. All animal studies comply with the ARRIVE guidelines. They were conducted in accordance with EU directive 2010/63/EU for animal experiments and approved by the Institutional Animal Care and Use Committee $\left(\right.$ CEA N $\left.{ }^{\circ} 121\right)$ and the French Ministry of Higher Education and Research (APAFIS \#02285.03). 
Preclinical surgical model. After a week of habituation, 48 Wistar rats (7 weeks old) were randomly assigned to a unoperated group (CTRL $n=8)$, or to RYGB $(n=13)$, OAGB-15 $(n=14)$ and OAGB-35 $(n=13)$ surgery.

Surgical models have already been described in detail in previous publications ${ }^{37,54}$ and are only briefly described below.

Gastric pouch construction was similar in OAGB and RYGB. After laparotomy, loose gastric connections to the liver and the spleen were released along the greater curvature, and the suspensory ligament supporting the upper fundus was severed. A $35 \mathrm{~mm}$ staple gun was applied at the junction between the glandular and non-glandular stomach to the left allowing resection of the forestomach. A second stapler using a thoraco-abdominal device $(3-3.5 \mathrm{~mm})$ was applied, parallel to the first stapling, on the right side of the gastroesophageal junction to exclude the antrum and a part of the body of the stomach. The two staple lines result in a $\sim 0.5 \mathrm{~cm}$ wide gastric pouch.

For rats operated on OAGB, the jejunum was anastomosed to the gastric pouch $15 \mathrm{~cm}(\mathrm{OAGB}-15)$ or $35 \mathrm{~cm}$ (OAGB-35) from the duodenojejunal angle with 7-0 isotactic polypropylene running sutures.

For rats operated on RYGB, the jejunum was transected $15 \mathrm{~cm}$ distally from the duodenojejunal angle. The Roux limb was anastomosed to the gastric pouch and the biliopancreatic limb was anastomosed $20 \mathrm{~cm}$ distal to the gastrojejunal anastomosis with 7-0 isotactic polypropylene running sutures.

The limb lengths chosen for the RYGB and OAGB-35 reproduce the ratio between the different segments commonly done in human procedures. OAGB-15 corresponds to an OAGB with a short BPL, inferior to $1 \mathrm{~m}$, in humans.

All rats were kept with free access to water during day 1 post-surgery. On day 2 and 3 post-surgery, rats had free access to a liquid diet, and on day 4 , they had access ad libitum to a normal diet. Unoperated rats followed the same post-operative nutrition protocol.

The overall survival rate was $82 \%$ with death (9/48) occurring in the immediate post-operative period. Out of the 9 deaths, 3 occurred in each group of gastric bypass.

Bile acid concentrations. After euthanasia, esophagi and stomachs were removed and flushed with $500 \mu \mathrm{L}$ of phosphate buffered saline (D-PBS) that were collected and stored at $-20^{\circ} \mathrm{C}$. Bile acids were extracted by solid-phase extraction and analyzed using high-performance liquid chromatography tandem mass spectrometry (HPLC MS/MS) as previously described ${ }^{55}$.

Calorimetric analyses. Macronutrient absorption was determined by fecal analyses as previously described ${ }^{37}$. Stools were collected within $24 \mathrm{~h}$ during the second postoperative week. Quantification of daily energetic loss $(\mathrm{kCal} / 24 \mathrm{~h})$ was determined by bomb calorimetry (PARR 1351 Bomb Calorimeter; Parr Instrument) and the daily caloric loss (\%) was expressed as a ratio of daily energetic loss on daily food intake.

Biochemical analyses. Blood samples (plasma) were collected during euthanasia 30 weeks after surgery. Samples were used for determination of concentrations of creatinine, urea, albumin, protein, calcium, phosphates, iron, ferritin, transferrin, triglycerides, total and HDL cholesterol, Non Esterified Fatty Acids (NEFA), Alanine Aminotransferase (ALAT) and Aspartate Aminotransferase (ASAT), using an automatic analyzer AU400 (Olympus Diagnostics, Rungis, France). Vitamin D was assayed, with IDS-iSYS 25 VitDS (Immunodiagnostic Systems Holdings PLC, UK) assay following manufacturer's instructions.

Histological analyses. After euthanasia, routine histology i.e. Hematoxylin-Eosin-Saffron (HES) and Alcian Blue-Periodic Acid Schiff (AB-PAS) were performed on formalin-fixed esophagus and gastro-jejunal anastomosis.

Parameters analyzed were the presence of dysplasia, metaplasia, and cancer. Presence of esophageal hyperpapillomatosis (EHP) and esophagitis as well as foveolar hyperplasia (FH) on gastric mucosa, a feature of reactive gastritis, were also studied. Esophagitis was defined by the association of basal cell hyperproliferation, fibrosis, hyper ortho-keratosis, immune cell infiltration and presence of EHP. FH was defined as the association of fibrosis, hyperproliferation of smooth muscle cells, and parietal cells. Slides were interpreted by two expert anatomopathologists $(\mathrm{AC}$ and $\mathrm{MH})$ blinded for the corresponding animal group.

Immunohistochemistry. MUC2, MUC5B and MUC4 protein expression was studied using an automated 8 immunostainer (ES, Ventana Medical System, France) with the following antibodies MUC2 (H300 rabbit, sc15334 Santa Cruz 1/200), MUC5B (mouse, EUMUC5B 1/50), and MUC4 (1G8 mouse, sc33654 Santa Cruz $1 / 50$ ). Positive controls were included by staining normal rat tissues known to express a protein of interest, and negative controls were run with D-PBS instead of primary antibodies.

Statistical analyses. Quantitative values are expressed as mean \pm SEM. Comparison between groups was conducted using ANOVA after log conversion or Kruskal Wallis non-parametric tests followed by Dunn's multiple comparison tests where appropriate. Kinetic studies were analyzed with a 2-way ANOVA followed by Tukey's multiple comparison tests where appropriate. Qualitative values were compared using Fisher exact test. Empirical power at the $5 \%$ level has been obtained by Monte-Carlo simulations using 500 replications. For contingency table, the observed table was used to estimate the proportion in each group. For quantitative data, a linear model adjusted on the observed data was used to obtain the mean by factor and the standard deviation.

All analyses were conducted with R version 3.6.1 (2019-07-05) or GraphPad prism 7.03.

$P<0.05$ was considered to be significant.

Received: 23 September 2019; Accepted: 15 April 2020;

Published online: 30 April 2020 


\section{References}

1. Jakobsen, G. S. et al. Association of Bariatric Surgery vs Medical Obesity Treatment With Long-term Medical Complications and Obesity-Related Comorbidities. Jama 319, 291-301 (2018).

2. Rutledge, R. The mini-gastric bypass: experience with the first 1,274 cases. Obes. Surg. 11, 276-280 (2001).

3. Victorzon, M. Single-anastomosis gastric bypass: better, faster, and safer? Scand. J. Surg. SJS Off. Organ Finn. Surg. Soc. Scand. Surg. Soc. 104, 48-53 (2015).

4. Lee, W.-J. et al. Laparoscopic Roux-en-Y versus mini-gastric bypass for the treatment of morbid obesity: a prospective randomized controlled clinical trial. Ann. Surg. 242, 20-28 (2005).

5. Reche, F., Mancini, A., Borel, A.-L. \& Faucheron, J.-L. Totally Robotic Reversal of Omega-Loop Gastric Bypass to Normal Anatomy. Obes. Surg. 26, 1994-1995 (2016).

6. Bruzzi, M. et al. Revisional single-anastomosis gastric bypass for a failed restrictive procedure: 5-year results. Surg. Obes. Relat. Dis. Off. J. Am. Soc. Bariatr. Surg. 12, 240-245 (2016).

7. Lee, W.-J. et al. Laparoscopic Roux-en-Y vs. mini-gastric bypass for the treatment of morbid obesity: a 10-year experience. Obes. Surg. 22, 1827-1834 (2012).

8. Abou Ghazaleh, R. et al. Is Mini-Gastric Bypass a Rational Approach for Type-2 Diabetes? Curr. Atheroscler. Rep 19, 51 (2017).

9. Quan, Y. et al. Efficacy of Laparoscopic Mini Gastric Bypass for Obesity and Type 2 Diabetes Mellitus: A Systematic Review and Meta-Analysis. Gastroenterol. Res. Pract 2015, 152852 (2015).

10. Bruzzi, M., Chevallier, J.-M. \& Czernichow, S. One-Anastomosis Gastric Bypass: Why Biliary Reflux Remains Controversial? Obes. Surg. 27, 545-547 (2017).

11. Pera, M. et al. Influence of esophagojejunostomy on the induction of adenocarcinoma of the distal esophagus in Sprague-Dawley rats by subcutaneous injection of 2,6-dimethylnitrosomorpholine. Cancer Res. 49, 6803-6808 (1989).

12. Levrat, M., Lambert, R. \& Kirshbaum, G. Esophagitis produced by reflux of duodenal contents in rats. Am. J. Dig. Dis. 7, 564-573 (1962).

13. Greene, C. L., Worrell, S. G. \& DeMeester, T. R. Rat Reflux Model of Esophageal Cancer and Its Implication in Human Disease. Ann. Surg. 262, 910-924 (2015).

14. Fein, M. et al. Duodenoesophageal reflux induces esophageal adenocarcinoma without exogenous carcinogen. J. Gastrointest. Surg. Off. J. Soc. Surg. Aliment. Tract 2, 260-268 (1998).

15. Csendes, A. et al. Latest results (12-21 years) of a prospective randomized study comparing Billroth II and Roux-en-Y anastomosis after a partial gastrectomy plus vagotomy in patients with duodenal ulcers. Ann. Surg. 249, 189-194 (2009).

16. Aya, M., Yashiro, M., Nishioka, N., Onoda, N. \& Hirakawa, K. Carcinogenesis in the remnant stomach following distal gastrectomy with billroth II reconstruction is associated with high-level microsatellite instability. Anticancer Res. 26, 1403-1411 (2006).

17. Li, H. H., Zhang, Q. Z. H., Xu, L. \& Hu, J. W. Clinical outcome of esophageal cancer after distal gastrectomy: a prospective study. Int. J. Surg. Lond. Engl. 6, 129-135 (2008).

18. Nehra, D., Howell, P., Pye, J. K. \& Beynon, J. Assessment of combined bile acid and pH profiles using an automated sampling device in gastro-oesophageal reflux disease. Br. J. Surg. 85, 134-137 (1998).

19. Kauer, W. K. et al. Composition and concentration of bile acid reflux into the esophagus of patients with gastroesophageal reflux disease. Surgery 122, 874-881 (1997).

20. Runkel, M., Pauthner, M. \& Runkel, N. The First Case Report of a Carcinoma of the Gastric Cardia (AEG II) After OAGB-MGB. Obes. Surg., https://doi.org/10.1007/s11695-019-04193-9 (2019).

21. Aggarwal, S., Bhambri, A., Singla, V., Dash, N. R. \& Sharma, A. Adenocarcinoma of oesophagus involving gastro-oesophageal junction following mini-gastric bypass/one anastomosis gastric bypass. J. Minimal Access Surg., https://doi.org/10.4103/jmas. JMAS_320_18(2019).

22. Renehan, A. G., Tyson, M., Egger, M., Heller, R. F. \& Zwahlen, M. Body-mass index and incidence of cancer: a systematic review and meta-analysis of prospective observational studies. Lancet Lond. Engl. 371, 569-578 (2008).

23. Duthie, S. J. Folate and cancer: how DNA damage, repair and methylation impact on colon carcinogenesis. J. Inherit. Metab. Dis. 34, 101-109 (2011)

24. Castiglioni, S. \& Maier, J. A. M. Magnesium and cancer: a dangerous liason. Magnes. Res 24, S92-100 (2011).

25. Sieg, J., Sieg, A., Dreyhaupt, J. \& Schmidt-Gayk, H. Insufficient vitamin D supply as a possible co-factor in colorectal carcinogenesis. Anticancer Res. 26, 2729-2733 (2006).

26. Arterburn, D. E. \& Courcoulas, A. P. Bariatric surgery for obesity and metabolic conditions in adults. BMJ 349, g3961 (2014).

27. Mohapatra, S., Gangadharan, K. \& Pitchumoni, C. S. Malnutrition in obesity before and after bariatric surgery. Dis. - Mon. DM, https://doi.org/10.1016/j.disamonth.2019.06.008 (2019).

28. Robert, M. et al. Efficacy and safety of one anastomosis gastric bypass versus Roux-en-Y gastric bypass for obesity (YOMEGA): a multicentre, randomised, open-label, non-inferiority trial. Lancet Lond. Engl. 393, 1299-1309 (2019).

29. Bétry, C. et al. Need for Intensive Nutrition Care After Bariatric Surgery. JPEN J. Parenter. Enteral Nutr. 41, 258-262 (2017).

30. Bruzzi, M. et al. Long-Term Evaluation of Biliary Reflux After Experimental One-Anastomosis Gastric Bypass in Rats. Obes. Surg. 27, 1119-1122 (2017)

31. Welbourn, R. et al. Bariatric Surgery Worldwide: Baseline Demographic Description and One-Year Outcomes from the Second IFSO Global Registry Report 2013-2015. Obes. Surg. 28, 313-322 (2018).

32. WHO|Life expectancy. WHO, http://www.who.int/gho/mortality_burden_disease/life_tables/situation_trends/en/.

33. Quinn, R. Comparing rat's to human's age: how old is my rat in people years? Nutr. Burbank Los Angel. Cty. Calif 21, 775-777 (2005).

34. Wild, C. P. \& Hardie, L. J. Reflux, Barrett's oesophagus and adenocarcinoma: burning questions. Nat. Rev. Cancer 3, 676-684 (2003).

35. Chevallier, J. M. et al. One thousand single anastomosis (omega loop) gastric bypasses to treat morbid obesity in a 7-year period: outcomes show few complications and good efficacy. Obes. Surg. 25, 951-958 (2015).

36. Saltzman, E. \& Karl, J. P. Nutrient deficiencies after gastric bypass surgery. Annu. Rev. Nutr. 33, 183-203 (2013).

37. Cavin, J.-B. et al. Malabsorption and intestinal adaptation after one anastomosis gastric bypass compared with Roux-en-Y gastric bypass in rats. Am. J. Physiol. Gastrointest. Liver Physiol. 311, G492-500 (2016).

38. Mahawar, K. K. et al. Impact of biliopancreatic limb length on severe protein-calorie malnutrition requiring revisional surgery after one anastomosis (mini) gastric bypass. J. Minimal Access Surg. 14, 37-43 (2018).

39. Gillard, L. et al. Enhanced Ghrelin Levels and Hypothalamic Orexigenic AgRP and NPY Neuropeptide Expression in Models of Jejuno-Colonic Short Bowel Syndrome. Sci. Rep 6, 28345 (2016).

40. Saeidi, N. et al. Reprogramming of intestinal glucose metabolism and glycemic control in rats after gastric bypass. Science 341, 406-410 (2013).

41. Moore, K. H., Barry, P., Burn, J. \& Falk, G. Adenocarcinoma of the rat esophagus in the presence of a proton pump inhibitor: a pilot study. Dis. Esophagus Off. J. Int. Soc. Dis. Esophagus 14, 17-22 (2001).

42. Nishijima, K. et al. Impact of the biliary diversion procedure on carcinogenesis in Barrett's esophagus surgically induced by duodenoesophageal reflux in rats. Ann. Surg. 240, 57-67 (2004).

43. M'Harzi, L. et al. Long-Term Evaluation of Biliary Reflux on Esogastric Mucosae after One-Anastomosis Gastric Bypass and Esojejunostomy in Rats. Obes. Surg., https://doi.org/10.1007/s11695-020-04521-4 (2020)

44. Mayne, S. T. \& Navarro, S. A. Diet, obesity and reflux in the etiology of adenocarcinomas of the esophagus and gastric cardia in humans. J. Nutr 132, 3467S-3470S (2002). 
45. Savarino, E., Marabotto, E. \& Savarino, V. Effects of bariatric surgery on the esophagus. Curr. Opin. Gastroenterol. 34, 243-248 (2018).

46. Weber, C. E., Kanani, Z., Schumm, M., Helm, M. \& Gould, J. C. Roux-en-Y gastric bypass as a salvage procedure in complicated patients with failed fundoplication(s). Surg. Endosc. 33, 738-744 (2019).

47. Kim, M. et al. Minimally invasive Roux-en-Y gastric bypass for fundoplication failure offers excellent gastroesophageal reflux control. Am. Surg. 80, 696-703 (2014).

48. Ireland, A. P. et al. Gastric juice protects against the development of esophageal adenocarcinoma in the rat. Ann. Surg. 224, 358-370, discussion 370-371 (1996).

49. Singh, M. et al. Weight loss can lead to resolution of gastroesophageal reflux disease symptoms: a prospective intervention trial. Obes. Silver Spring Md 21, 284-290 (2013).

50. De Pergola, G. \& Silvestris, F. Obesity as a major risk factor for cancer. J. Obes. 2013, 291546 (2013).

51. Thrift, A. P. et al. Obesity and risk of esophageal adenocarcinoma and Barrett's esophagus: a Mendelian randomization study. J. Natl. Cancer Inst. 106 (2014).

52. Sebastianelli, L. et al. Systematic Endoscopy 5 Years After Sleeve Gastrectomy Results in a High Rate of Barrett's Esophagus: Results of a Multicenter Study. Obes. Surg. 29, 1462-1469 (2019).

53. Genco, A. et al. Gastroesophageal reflux disease and Barrett's esophagus after laparoscopic sleeve gastrectomy: a possible, underestimated long-term complication. Surg. Obes. Relat. Dis. Off. J. Am. Soc. Bariatr. Surg. 13, 568-574 (2017).

54. Siebert, M. A. et al. One-anastomosis Gastric Bypass (OAGB) in Rats. J. Vis. Exp. JoVE, https://doi.org/10.3791/58776 (2018).

55. Duboc, H. et al. Roux-en-Y Gastric-Bypass and sleeve gastrectomy induces specific shifts of the gut microbiota without altering the metabolism of bile acids in the intestinal lumen. Int. J. Obes. 2005 43, 428-431 (2019).

\section{Acknowledgements}

We thank the group of Prof. N. Kapel of the Department of Functional Coprology, APHP, and Chelsea Chachoute for stool analyses. We thank N Sorhaindo, Biochemistry Platform CRI, for plasma samples analyses, Dr. T Dupré for Vitamin D assay, O Thibaudeau and S. Ameur, Morphology Platform, Bichat Hospital, for help in histologic experiments. We thank M. Y from IDS ImmunoDiagnostic System, Paris, France for providing free Vitamin D assays. M. Le Gall is grateful to Drs. L. Arnaud, J. Le Beyec, and S. Ledoux for supportive advice and comments along this study and on the manuscript. MS and LRP received funding from Fondation de la Recherche Médicale (FRM) and Association Française de Chirurgie (AFC). MLG is supported by a grant from Société Francophone du Diabète, Institut Benjamin Delessert and Fondation de l'Avenir. Authors are supported by Inserm, Université de Paris and AP-HP.

\section{Author contributions}

M.L.G. and A.B. designed the experiments, acquired and interpreted data. M.S. and L.R.P. performed the surgeries, acquired and interpreted data. M.H. and A.C. performed and interpreted the immunohistochemistry analyses. N.D.N. and N.K. performed and interpreted the coprology analyses. B.D. and N.J. performed and interpreted immunohistology analyses. L.H., H.D. and D.R. performed and interpreted the BA analyses. G.N. performed most of the statistical analyses. J.M.C., S.M. and H.D. contributed to the design of the experiment, the interpretation of the data and, revised the manuscript critically for important intellectual content. M.S. and M.L.G. wrote the article with critical revisions for important intellectual content from A.B. and N.D.N. edited the English of the manuscript. All authors discussed the results, commented on the manuscript and gave final approval of the version to be submitted.

\section{Competing interests}

The authors declare no competing interests.

\section{Additional information}

Supplementary information is available for this paper at https://doi.org/10.1038/s41598-020-64425-2.

Correspondence and requests for materials should be addressed to M.L.G.

Reprints and permissions information is available at www.nature.com/reprints.

Publisher's note Springer Nature remains neutral with regard to jurisdictional claims in published maps and institutional affiliations.

Open Access This article is licensed under a Creative Commons Attribution 4.0 International License, which permits use, sharing, adaptation, distribution and reproduction in any medium or format, as long as you give appropriate credit to the original author(s) and the source, provide a link to the Creative Commons license, and indicate if changes were made. The images or other third party material in this article are included in the article's Creative Commons license, unless indicated otherwise in a credit line to the material. If material is not included in the article's Creative Commons license and your intended use is not permitted by statutory regulation or exceeds the permitted use, you will need to obtain permission directly from the copyright holder. To view a copy of this license, visit http://creativecommons.org/licenses/by/4.0/.

(c) The Author(s) 2020 